\title{
miR-146a-5p plays an essential role in the aberrant epithelial-fibroblast cross-talk in COPD
}

\author{
Emmanuel T. Osei ${ }^{1,2,3}$, Laura Florez-Sampedro ${ }^{2,4}$, Hataitip Tasena ${ }^{1,2}$, \\ Alen Faiz ${ }^{1,2}$, Jacobien A. Noordhoek ${ }^{1,2,5}$, Wim Timens ${ }^{1,2}$, Dirkje S. Postma ${ }^{2,5}$, \\ Tillie L. Hackett ${ }^{3}$, Irene H. Heijink ${ }^{1,2,5,6}$ and Corry-Anke Brandsma 1,2,6
}

Affiliations: 'Dept of Pathology and Medical Biology, University of Groningen, University Medical Center Groningen, Groningen, The Netherlands. ${ }^{2}$ GRIAC Research Institute, University of Groningen, University Medical Center Groningen, Groningen, The Netherlands. ${ }^{3}$ Centre for Heart and Lung Innovation, University of British Columbia, Vancouver, BC, Canada. ${ }^{4}$ Dept of Pharmacokinetics, Toxicology and Targeting, University of Groningen, Groningen, The Netherlands. ${ }^{5}$ Dept of Pulmonology, University of Groningen, University Medical Center Groningen, Groningen, The Netherlands. ${ }^{6}$ These two authors contributed equally to this work.

Correspondence: Emmanuel T. Osei, Centre for Heart Lung Innovation, University of British Columbia and St Paul's Hospital, 1081 Burrard Street, Room 166, Burrard Building, Vancouver, BC V6Z 1Y6, Canada.

E-mail: emmanuel.oseiQhli.ubc.ca

@ERSpublications

Defective upregulation of miR-146a-5p in lung fibroblasts leads to fibroblast-mediated inflammation in COPD http://ow.ly/3awN308JqRH

Cite this article as: Osei ET, Florez-Sampedro L, Tasena H, et al. miR-146a-5p plays an essential role in the aberrant epithelial-fibroblast cross-talk in COPD. Eur Respir J 2017; 49: 1602538 [https://doi.org/ 10.1183/13993003.02538-2016].

ABSTRACT We previously reported that epithelial-derived interleukin (IL)- $1 \alpha$ drives fibroblast-derived inflammation in the lung epithelial-mesenchymal trophic unit. Since miR-146a-5p has been shown to negatively regulate IL-1 signalling, we investigated the role of miR-146a-5p in the regulation of IL- $1 \alpha$-driven inflammation in chronic obstructive pulmonary disease (COPD).

Human bronchial epithelial (16HBE14o-) cells were co-cultured with control and COPD-derived primary human lung fibroblasts (PHLFs), and miR-146a-5p expression was assessed with and without IL- $1 \alpha$ neutralising antibody. Genomic DNA was assessed for the presence of the single nucleotide polymorphism (SNP) rs2910164. miR-146a-5p mimics were used for overexpression studies to assess IL- $1 \alpha$-induced signalling and IL- 8 production by PHLFs.

Co-culture of PHLFs with airway epithelial cells significantly increased the expression of miR-146a-5p and this induction was dependent on epithelial-derived IL- $1 \alpha$. miR-146a-5p overexpression decreased IL- $1 \alpha$-induced IL- 8 secretion in PHLFs via downregulation of IL- 1 receptor-associated kinase- 1 . In COPD PHLFs, the induction of miR-146a-5p was significantly less compared with controls and was associated with the SNP rs2910164 (GG allele) in the miR-146a-5p gene.

Our results suggest that induction of miR-146a-5p is involved in epithelial-fibroblast communication in the lungs and negatively regulates epithelial-derived IL- $1 \alpha$ induction of IL- 8 by fibroblasts. The decreased levels of miR-146a-5p in COPD fibroblasts may induce a more pro-inflammatory phenotype, contributing to chronic inflammation in COPD.

This article has supplementary material available from erj.ersjournals.com

Received: May 102016 | Accepted after revision: Jan 292017

Support statement: This study was funded by Jan Kornelis de Cock Stichting and Stichting Astma Bestrijding. Funding information for this article has been deposited with the Crossref Funder Registry.

Conflict of interest: Disclosures can be found alongside this article at erj.ersjournals.com

Copyright OERS 2017 


\section{Introduction}

Chronic obstructive pulmonary disease (COPD) is a progressive disease in which chronic neutrophilic inflammation is associated with destruction of the lung parenchyma (emphysema) and small airway disease, which both contribute to airflow limitation and lung function decline [1]. The inhalation of noxious particles, specifically from cigarette smoke, is the most common risk factor for COPD and smoking is known to induce the recruitment of neutrophils $[1,2]$. However, not all smokers develop COPD, indicating a genetic susceptibility to the disease process [3]. The inconsistency in replicating target genes related to COPD in different study populations points to an important role of epigenetic regulation [4]. Epigenetic mediators such as microRNAs (miRNAs) can regulate the transcriptional activity of various genes involved in lung function and inflammation that are thought to be involved in the pathogenesis of COPD [5].

miRNAs are small noncoding RNAs of approximately 19-25 nucleotides that cause post-transcriptional gene repression by increasing mRNA degradation or by inhibiting protein translation of specific mRNA targets [6]. They are involved in various biological processes and alterations in their expression can result in pathological conditions, including pulmonary diseases [7]. We recently provided an up-to-date review of studies showing the role of miRNA dysregulation in COPD and how it is associated with the various features of COPD [5]. In particular, it has been shown that exposure to cigarette smoke alone can change miRNA expression in the lungs [8]. Several studies have shown that various miRNAs are differentially expressed in whole lung tissue, serum and/or sputum of smoking COPD patients compared with smokers without COPD [9-12]. Of these, miR-146a-5p has been shown to regulate the release of interleukin (IL)-1-induced inflammatory mediators from pulmonary epithelial cells, including the neutrophil chemoattractant IL-8 [13]. We recently showed in a co-culture model that cigarette smoke extract (CSE)-induced IL-1 $\alpha$ expression is higher in airway epithelial cells (AECs) from COPD patients compared with control-derived epithelial cells [14]. Furthermore, the higher levels of epithelial IL-1 $\alpha$ induce a stronger release of the pro-inflammatory cytokines including IL-8 from lung fibroblasts [14]. Interestingly, others have shown that when lung fibroblasts are stimulated with IL-1 $\beta$ and tumour necrosis factor (TNF)- $\alpha$, miR-146a-5p expression is induced to a lesser extent in lung fibroblasts from COPD patients when compared with control fibroblasts [12].

In this study, we hypothesised that a failure to upregulate miR-146a-5p in lung fibroblasts contributes to the disturbed communication within the epithelial-mesenchymal trophic unit in COPD, leading to increased inflammation in the disease. Therefore, we studied the expression of miR-146a-5p in COPD and non-COPD control-derived primary human lung fibroblasts (PHLFs) in our co-culture model, and conducted functional assays to investigate the mechanism of regulation of pro-inflammatory activity by miR-146a-5p in pulmonary fibroblasts.

\section{Materials and methods}

\section{Subjects and cell culture conditions}

The human bronchial epithelial cell line 16HBE14o- was kindly donated by Dr D.C. Gruenert (University of California, San Francisco, CA, USA) and cultured in Eagle's minimal essential medium (EMEM)/10\% fetal calf serum (FCS; Lonza/BioWhittaker, Verviers, Belgium) on collagen/bovine serum albumin-coated flasks as previously described [15].

Fetal lung fibroblast cells (MRC-5; BioWhittaker, Walkersville, MD, USA) were cultured in EMEM/10\% FCS on 24-well plates before experiments. PHLFs were isolated from peripheral parenchymal lung tissue of eight non-COPD control donors undergoing tumour resection surgery and 12 COPD patients with severe disease undergoing lung transplantation using the explant technique as previously described [16, 17]. PHLFs from controls were isolated from histologically normal tissue taken as far away as possible from the tumour. The tissue was checked for cancer or other pathology by an experienced pathologist, and found to be cancer-free and showing no other pathology. Clinical information on the COPD patients and non-COPD control subjects is presented in table 1 . The study protocol for this project was consistent with the Research Code of the University Medical Center Groningen (www.rug.nl/umcg/onderzoek/ researchcode/index) and national ethical and professional guidelines (www.federa.org). PHLFs were cultured in Ham's-F12 medium/10\% FCS (Lonza) on 24-well culture plates before experiments.

\section{Co-culture model}

We used 16HBE14o- and MRC-5 co-cultures for the mechanistic studies. 16HBE14o- cells were co-cultured with COPD and control-derived PHLFs to determine the disease-specific effects.

Briefly, 16HBE14o- cells were plated on coated $0.4-\mu \mathrm{M}$ pore $6.5-\mathrm{mm}$ transwell membranes (Costar; Corning, New York, NY, USA), while fibroblasts were cultured separately on a 24-well plate. After a confluent layer was obtained for both cell types, the transwell with 16HBE14o- cells (upper compartment) was placed in co-culture with lung fibroblasts in the 24-well plate (lower compartment) and left for $72 \mathrm{~h}$ in 
EMEM/10\% FCS or Ham's-F12 medium/10\% FCS (Lonza) for co-culture with MRC-5 cells or PHLFs, respectively [14]. Before experimentation, cells were serum-deprived overnight.

\section{Cigarette smoke extract preparation}

CSE experiments were performed in serum-free, hormone-supplemented bronchial epithelial basal medium media (Lonza, Basel, Switzerland). CSE was prepared before each experiment by bubbling two filterless cigarettes through $25 \mathrm{~mL}$ media to make 100\% CSE, which was then diluted to $20 \%$ CSE as previously described [18].

\section{Conditioned medium and neutralising antibody experiments}

$16 \mathrm{HBE} 14 \mathrm{o}$ - cells were serum-deprived overnight and stimulated for $6 \mathrm{~h}$ with or without $20 \%$ CSE, which we have previously reported did not affect cell viability [14]. After stimulation, the CSE was thoroughly washed off and CSE-free conditioned medium was collected after a further $24 \mathrm{~h}$ incubation. CSE-free conditioned medium was pre-incubated for $1 \mathrm{~h}$ with or without $4 \mu \mathrm{g} \cdot \mathrm{mL}^{-1} \mathrm{IL}-1 \alpha$ neutralising antibody (AB-200-NA, MAB601; R\&D Systems, Abingdon, UK) and was used to stimulate serum-deprived fibroblasts for $24 \mathrm{~h}$. For experiments with recombinant human (rh) IL-1 $\alpha$ (R\&D Systems), a $1 \mathrm{ng} \cdot \mathrm{mL}^{-1}$ concentration was chosen as this is comparable to levels previously reported in sputum of COPD patients [19]. Cell-free supernatants were collected and analysed by ELISA, and cell lysates were harvested with TRI Reagent (Molecular Research Center, Cincinnati, OH, USA) for RNA isolation.

miR-146a-5p mimic transfection

MRC-5 fibroblasts were transfected with the miR-146a-5p mimic at $25 \mathrm{nM}$ (MirVana miRNA mimic, assay ID: MC10722; Applied Biosystems, Carlsbad, CA, USA) and scrambled small RNA at $25 \mathrm{nM}$ (AllStars Negative Control siRNA; Qiagen, Hilden, Germany) as a nontargeting control to assess the effects of miR-146a-5p induction on IL-1 signalling in lung fibroblasts. miR-146a-5p expression was analysed by

TABLE 1 Characteristics of chronic obstructive pulmonary disease (COPD) patients and non-COPD controls from whom primary human lung fibroblasts (PHLFs) were obtained

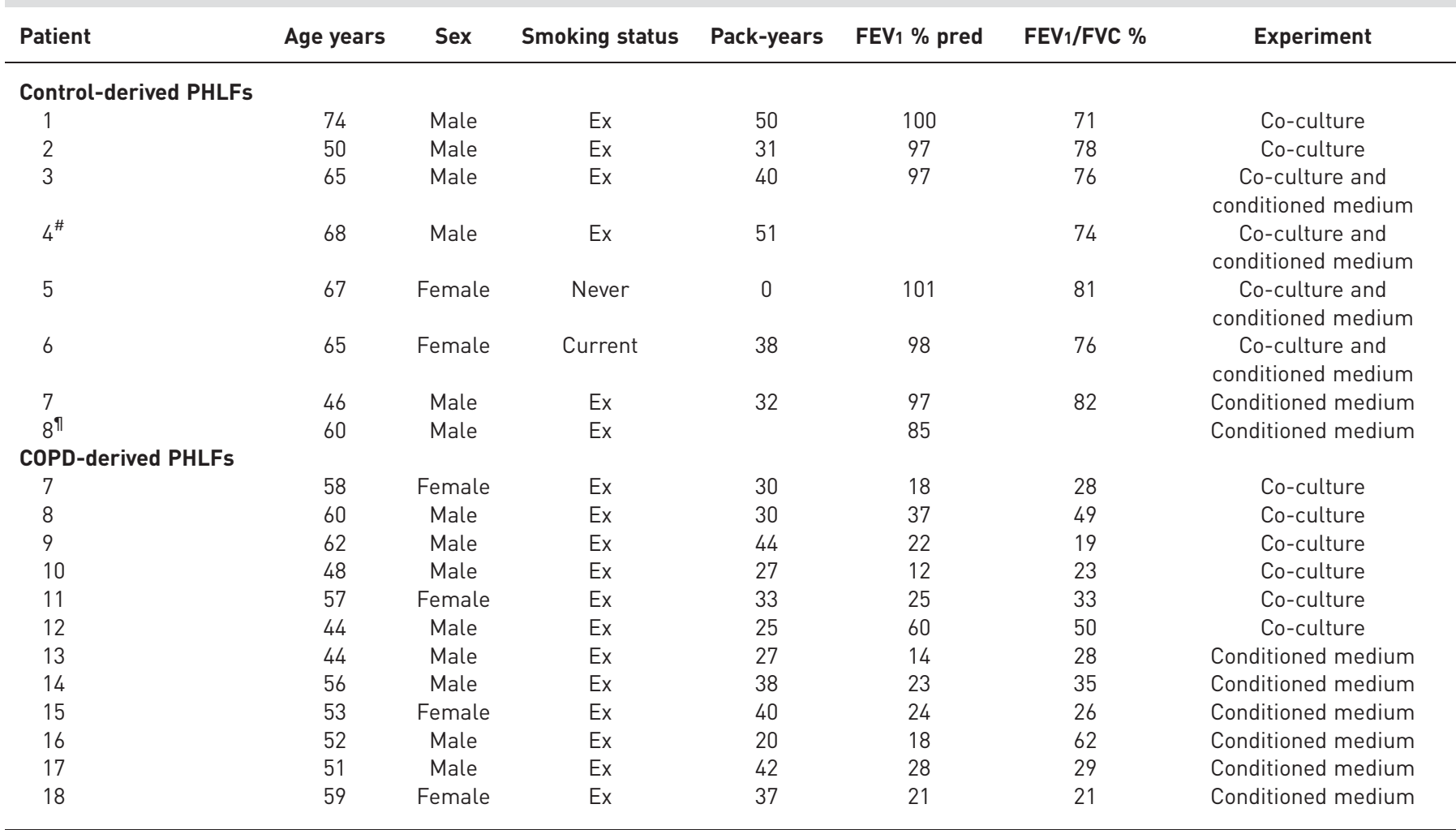

All COPD donors were on inhaled or oral steroids before transplantation. FEV1: forced expiratory volume in $1 \mathrm{~s}$; FVC: forced vital capacity. ${ }^{\#}$ : FEV $1 \%$ pred not available; " : pack-years and FEV $1 / F V C$ not available. There was a significant difference in FEV $1 \%$ pred ( $\left.p=0.04\right)$ and $\mathrm{FEV}_{1} / \mathrm{FVC} \%(p=0.04)$, as well as in age between the COPD patients and controls, with the COPD patients being slightly younger ( $p=0.03$ ). There was no significant difference in pack-years between the groups. 
quantitative PCR, protein lysates were assessed by Western blot and IL-8 concentrations were determined by ELISA (R\&D Systems) in the cell-free supernatant as described in the supplementary material.

\section{Single nucleotide polymorphism genotyping}

Genomic DNA for genotyping was extracted from lung tissue of PHLF donors and assessed for the presence of the single nucleotide polymorphism (SNP) rs2910164. A detailed description is available in the supplementary material.

\section{Statistical analysis}

SPSS Statistics version 23 (IBM, Armonk, NY, USA) was used for data analyses. Differences between COPD patients and non-COPD control subjects were analysed with a Mann-Whitney U-test. Differences between treatments within a group were analysed using paired t-tests for the cell lines and Wilcoxon signed-rank tests for the primary cells. $\mathrm{p}<0.05$ was considered statistically significant.

\section{Results}

Epithelium-derived IL-1 $\alpha$ is responsible for the increased miR-146a-5p expression in lung fibroblasts

As our previous study showed that IL- $1 \alpha$ derived from the airway epithelium (primary and 16HBE140cells) is an important regulator of pulmonary fibroblast-derived inflammation [14], we first determined
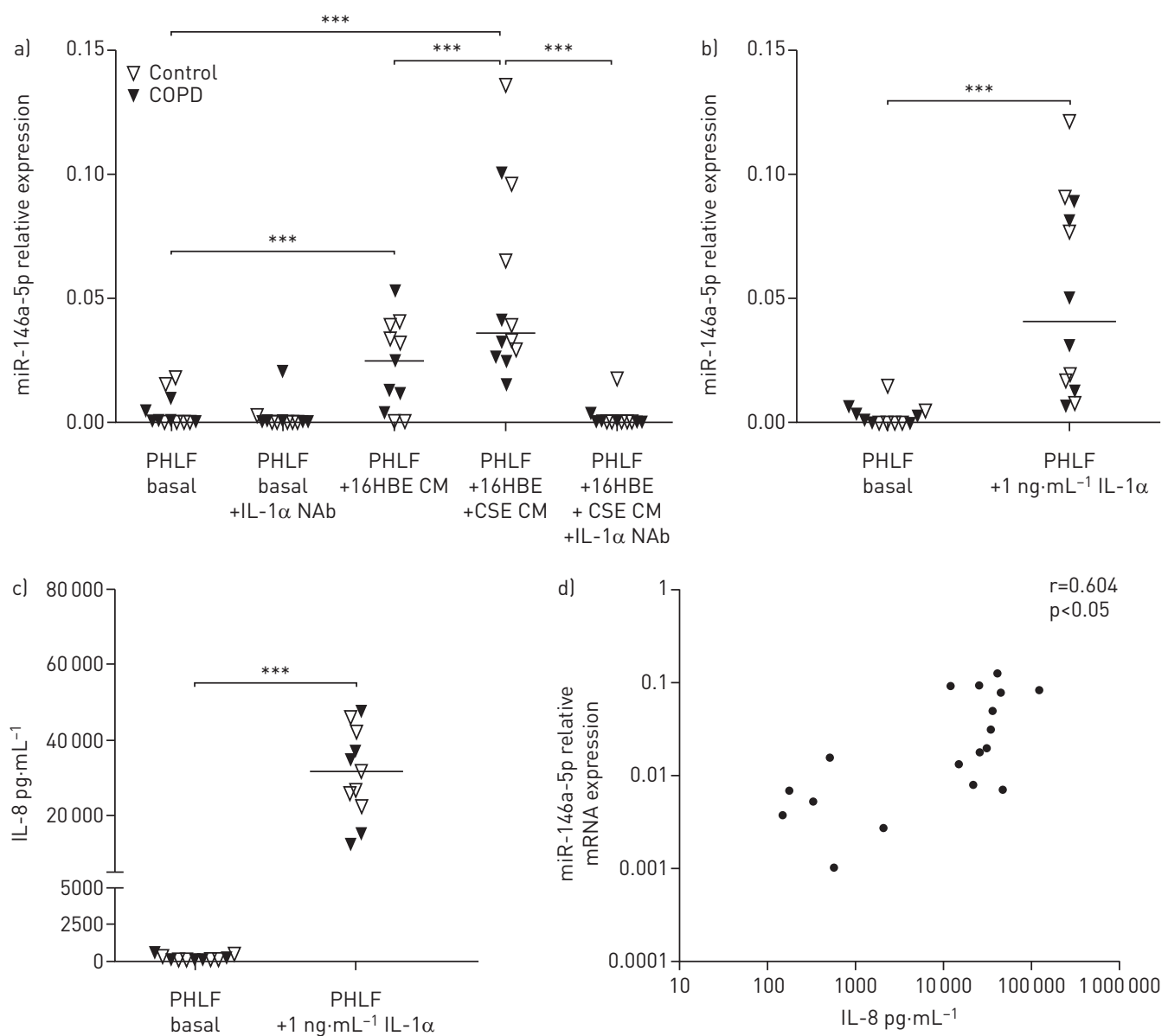

FIGURE 1 Epithelial-derived interleukin (IL)-1 $\alpha$ is responsible for increased miR-146a-5p expression in lung fibroblasts. Primary human lung fibroblasts (PHLFs) from control donors $(n=6)$ and chronic obstructive pulmonary disease (COPD) patients $(n=6)$ were grown to confluence and serum-deprived overnight. a) miR-146a-5p expression in PHLFs after stimulation with conditioned medium (CM) from 16HBE140- (16HBE) cells pre-treated with or without cigarette smoke extract (CSE) in the presence or absence of $4 \mu \mathrm{g} \cdot \mathrm{mL}^{-1} \mathrm{IL}-1 \alpha$ neutralising antibody (NAb). b) miR-146a-5p expression in serum-deprived PHLFs after stimulation with/without $1 \mathrm{ng} \cdot \mathrm{mL}^{-1}$ recombinant human IL-1 $\alpha$ for $24 \mathrm{~h}$. miR-146a-5p expression levels were related to the housekeeping noncoding RNU48, expressed as $2^{-\Delta C_{t}}$. c) IL-8 concentration released from PHLF. Medians are indicated. ***: $p<0.001$, between the indicated values. d) Correlation of IL-8 concentration released from PHLFs to miR-146a-5p expression in PHLFs after IL-1 $\alpha$ stimulation. Data are shown on a log scale. 
whether epithelial-derived IL- $1 \alpha$ causes an induction of miR-146a-5p in lung fibroblasts. We stimulated PHLFs from non-COPD controls and COPD patients with 16HBE14o- conditioned medium in which IL- $1 \alpha$ levels had been previously determined [14] in the presence or absence of the IL- $1 \alpha$ neutralising antibody. We found that the expression of miR-146a-5p was increased in PHLFs treated with conditioned media from 16HBE14o- cells and this was further enhanced by pre-stimulation of the 16HBE14o- cells with CSE (figure 1a). Furthermore, the induction of miR-146a-5p was completely abrogated by the addition of IL- $1 \alpha$ neutralising antibody (figure 1a). We also assessed the direct effects of rhIL- $1 \alpha$ stimulation on miR-146a-5p expression in PHLFs and found, as with the 16HBE14o- cell conditioned media, a significant increase in miR-146a-5p expression in PHLFs (figure 1b). Lastly, stimulation with $1 \mathrm{ng} \cdot \mathrm{mL}^{-1}$ (figure 1c) or $0.01 \mathrm{ng} \cdot \mathrm{mL}^{-1}$ (supplementary figure S1) rhIL-1 $\alpha$ led to a significant release of IL-8 concentration from PHLFs (figure 1c), which significantly correlated with the increased expression of miR-146a-5p in PHLFs (figure 1d). Neither the expression level of miR-146a-5p nor the release of IL-8 was significantly different between COPD and control-derived PHLFs after 16HBE14o- conditioned medium or rhIL- $1 \alpha$ stimulations.

miR-146a-5p expression is decreased in PHLFs from COPD patients in co-culture with 16HBE140- cells

We have previously shown in our co-culture model that COPD-derived AECs, through higher induction of IL- $1 \alpha$, induce lung fibroblasts to be more pro-inflammatory [14]. Hence, we were interested in the regulation of miR-146a-5p expression in control and COPD-derived PHLFs. In our co-culture model, 16HBE14o- cells significantly upregulated miR-146a-5p expression in PHLFs from both control and COPD donors. Interestingly, there was a significantly lower induction of miR-146a-5p in COPD-derived PHLFs compared with those from control donors upon co-culture with 16HBE14o- cells (figure 2).

\section{Lower induction of miR-146a-5p in COPD fibroblasts is associated with the single nucleotide polymorphism rs2910164}

To explain the difference in miR-146a-5p induction between COPD and control fibroblasts, we considered two possibilities. 1) A difference in expression of RelB, a member of the NF- $\kappa \mathrm{B}$ family of transcription factors, that has been shown to regulate miR-146a-5p in mouse lung fibroblasts [20]. 2) A difference in the presence of the SNP rs2910164 in the primary miR-146a-5p sequence as this SNP has been shown to cause a reduction in the expression of mature miR-146a-5p [21].

1) We examined the expression of RelB in our PHLFs from the co-culture model, and we found no significant difference in mRNA expression of RelB between control and COPD-derived PHLFs (figure 3a). 2) We compared the genotypes for the rs2910164 SNP of the PHLFs used in our co-culture experiments and found that donors with the GG genotype had a lower miR-146a-5p induction after co-culture than those with the CG genotype (figure 3b). Importantly the donors with the GG genotype were all, except one, COPD patients.

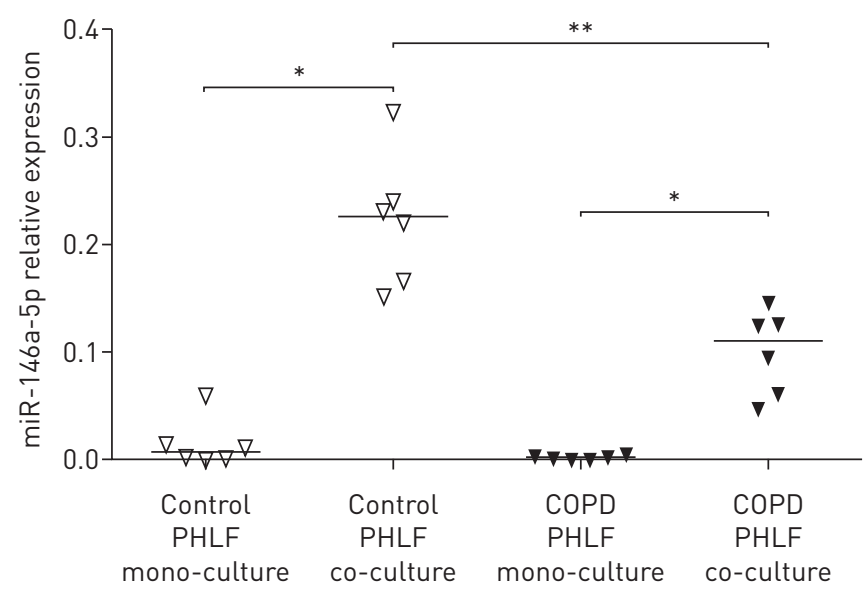

FIGURE 2 Expression of miR-146a-5p in primary human lung fibroblasts (PHLFs) upon co-culture with 16HBE140- cells. PHLFs from control donors or chronic obstructive pulmonary disease (COPD) patients were cultured alone or co-cultured with $16 \mathrm{HBE} 140$ - cells for $72 \mathrm{~h}$ after which they were serum-deprived. miR-146a-5p expression in PHLFs from control donors and COPD patients in mono-culture and co-culture with 16HBE140- cells was related to the housekeeping noncoding RNU48, expressed as $2^{-\Delta C_{t}}$. Medians are indicated. ${ }^{*}$ : $p<0.05 ;{ }^{* *}$ : $p<0.01$, between the indicated values. 
miR-146a-5p overexpression has anti-inflammatory effects on lung fibroblasts

miR-146a-5p has been reported to exert anti-inflammatory properties by targeting the proteins of IL-1 receptor-associated kinase (IRAK)-1 and TNF receptor-associated factor (TRAF)-6, which are key downstream mediators in the cellular response to IL-1 [22]. Hence, we were interested in examining the anti-inflammatory mechanism of miR-146a-5p in human lung fibroblasts. For overexpression of miR-146a-5p experiments we used a human lung fetal fibroblast cell line (MRC-5). First, we examined the expression of miR-146a-5p in MRC-5 fibroblasts co-cultured with 16HBE14o- cells and found a similar induction of miR-146a-5p in MRC-5 fibroblasts upon co-culture (figure 4a) as in the PHLFs. Next, we successfully overexpressed miR-146a-5p levels in MRC-5 fibroblasts by treatment with the miR-146a-5p mimic compared with the scrambled nontargeting control mimic (figure $4 \mathrm{~b}$ ). We then assessed the protein expression levels of IRAK-1 and TRAF- 6 in MRC-5 fibroblasts after overexpressing miR-146a-5p. We found the protein expression of IRAK-1 was significantly reduced after miR-146a-5p overexpression compared with the scrambled control, but TRAF- 6 was unaffected (figure $4 \mathrm{c}$ and $\mathrm{d}$ ). This indicates that miR-146a-5p indeed regulates IL-1 receptor downstream signalling in human lung fibroblasts.

To determine the role of miR-146a-5p in the suppression of IL- $1 \alpha$-induced IL-8 release, we treated MRC-5 fibroblasts with rhIL- $1 \alpha$ after miR-146a-5p overexpression (figure 5a). Here, we found a significant decrease of IL-8 release from MRC-5 fibroblasts when miR-146a-5p was overexpressed compared with cells treated with the scrambled control (figure 5b). Taken together, our data show that miR-146a-5p regulates IL-1 $\alpha$-induced pro-inflammatory responses in lung fibroblasts (figure 6).

\section{Discussion}

We investigated the role of miR-146a-5p in aberrant IL- $1 \alpha$ signalling between the airway epithelium and lung fibroblasts in COPD. We found that co-culture of PHLFs with AECs significantly increases the expression of miR-146a-5p, which is completely dependent on epithelial-derived IL-1 $\alpha$. We demonstrate that miR-146a-5p expression has an anti-inflammatory role, by downregulating the expression of IRAK-1, which is downstream of the IL-1 pathway, and subsequently reduces IL- 8 release from lung fibroblasts. Furthermore, we show that the induction of miR-146a-5p is significantly less in COPD fibroblasts and this was associated with the SNP rs2910164 (GG allele) in the miR-146a-5p gene.

miR-146a-5p has been well studied as a regulator of cellular function in both innate and adaptive immunity [23]. Specifically, miR-146a-5p has been suggested to target various inflammatory pathways including Toll-like receptor and IL-1 receptor signalling $[23,24]$. Overexpressing miR-146a-5p in the liver prevents the release of pro-inflammatory cytokines and protects mice from ischaemia-reperfusion injury by targeting and reducing the protein expression of IRAK-1 and TRAF-6, which are downstream of the IL-1 pathway [22]. Perry et al. [13] showed that miR-146a-5p overexpression reduces IL-1 $\beta$-induced IL-8 production in mono-cultures of alveolar epithelial cells. Additionally, BнAuмiк et al. [25] found a high expression of miR-146a-5p in senescent human neonatal foreskin fibroblasts compared with quiescent cells. This high
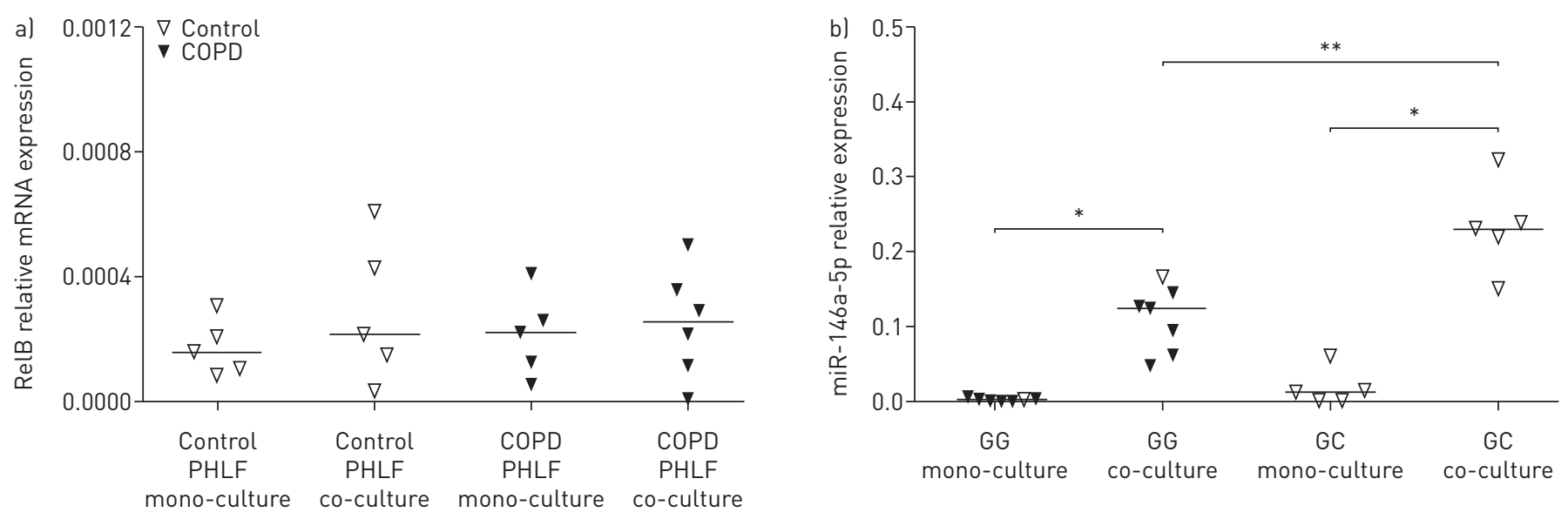

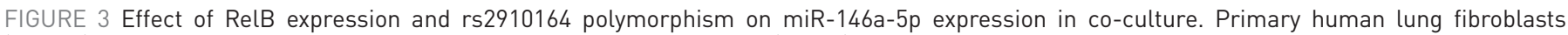
(PHLFs) from control donors or chronic obstructive pulmonary disease (COPD) patients were cultured alone or co-cultured with 16HBE140- cells for $72 \mathrm{~h}$ after which they were serum-deprived. a) RelB expression in PHLFs from control donors and COPD patients in mono-culture and co-culture with 16HBE140- cells. b) Effect of the single nucleotide polymorphism rs2910164 on miR-146a-5p expression in PHLFs before and after

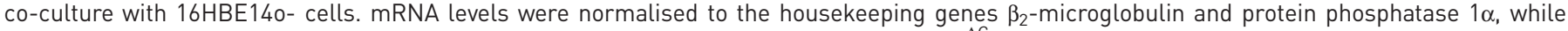

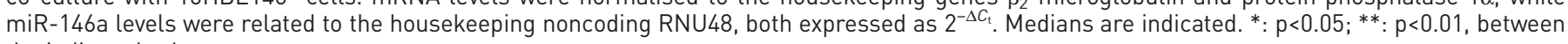
the indicated values. 

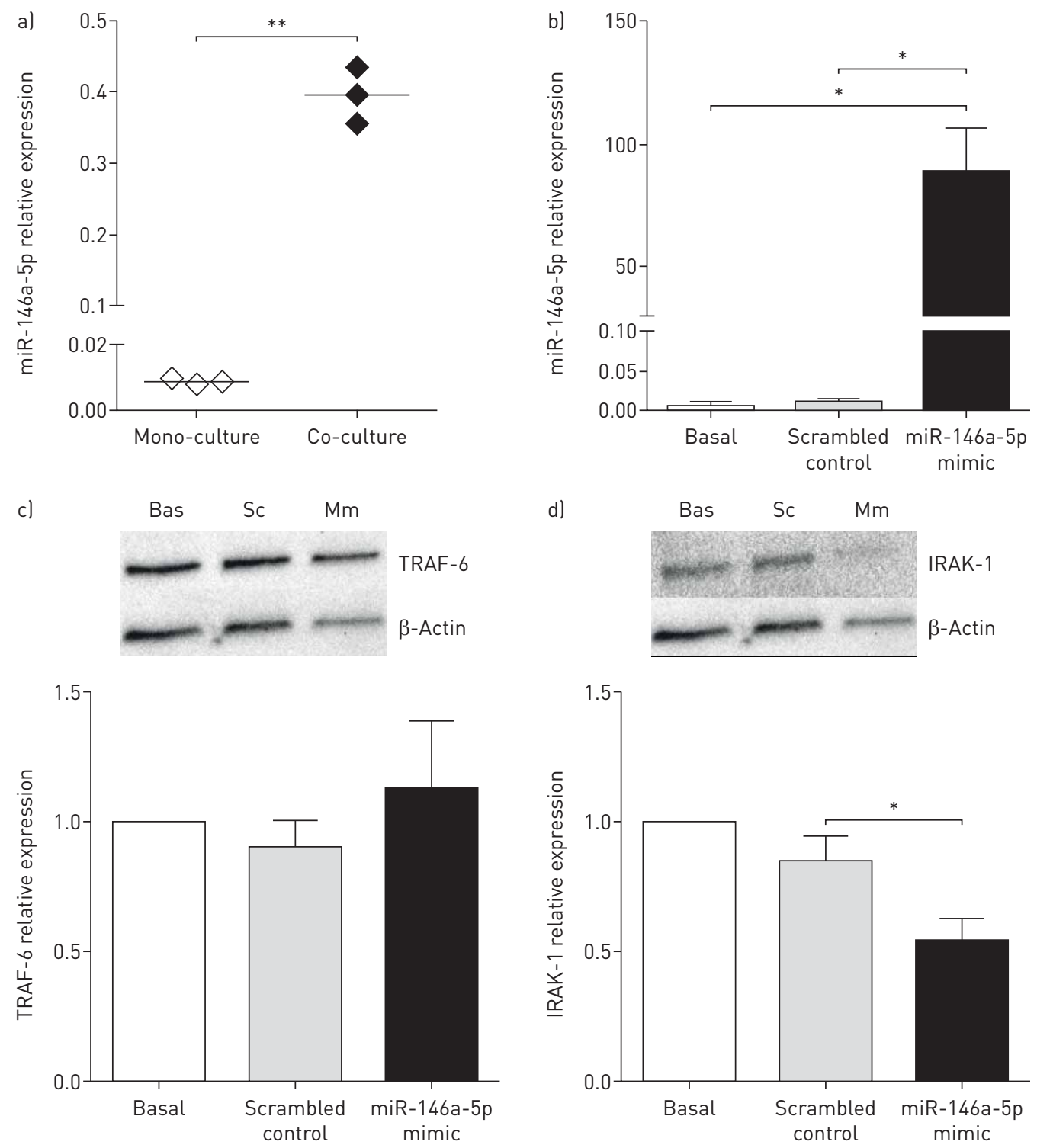

FIGURE 4 miR-146a-5p has anti-inflammatory effects in lung fibroblasts. a) MRC-5 fibroblasts were cultured alone or co-cultured with 16HBE140- cells. miR-146a-5p expression in the fibroblasts was related to the housekeeping noncoding RNU48, expressed as $2^{-\Delta C_{t}}$. Medians are indicated. b) MRC- 5 fibroblasts were seeded and immediately transfected with $25 \mathrm{nM}$ miR-146a-5p mimic or the scrambled control for $48 \mathrm{~h}$. miR-146a-5p expression in the fibroblasts was related to the housekeeping noncoding RNU48, expressed as $2^{-\Delta C_{t}}$. Data are presented as mean \pm SEM ( $n=3$ or 4 independent experiments). c) Tumour necrosis factor receptor-associated factor (TRAF)- 6 and d) interleukin-1 receptor-associated kinase (IRAK)-1 protein expression with representative blots and densitometry in MRC-5 fibroblasts after transfection with miR-146a-5p mimic (Mm) or scrambled control (Sc). Bas: basal. $\beta$-Actin was used as the loading control for protein expression. Data are presented as mean \pm SEM ( $n=3$ or 4 independent experiments). *: $p<0.05 ;{ }^{* *}: p<0.01$, between the indicated values.

expression was shown to reflect a negative feedback mechanism that modulates the secretion of IL- $1 \alpha$-induced IL- 6 and IL- 8 release due to a robust senescent-associated secretory phenotype activity in fibroblasts [25]. In line with our present study, this effect was linked to the inhibition of IRAK-1, but not TRAF-6 [25]. This finding is particularly important since senescence of various cell types such as epithelial cells and fibroblasts in the lung has been shown to contribute to COPD pathogenesis [26].

IL- $1 \alpha$ is an important driver of innate immune responses [27]. This cytokine is constitutively present in the lung epithelium as part of the immune defence against inhaled particles and is responsible for the release of chemokines, such as IL-8, responsible for neutrophilic recruitment $[27,28]$. In COPD, there is an increased release of IL- $1 \alpha$ as indicated by the increased levels in sputum and bronchoalveolar lavage fluid compared with control subjects [29]. We have previously shown that exposure to CSE induces a stronger expression of IL- $1 \alpha$ in airway epithelium from COPD patients compared with controls, leading to 

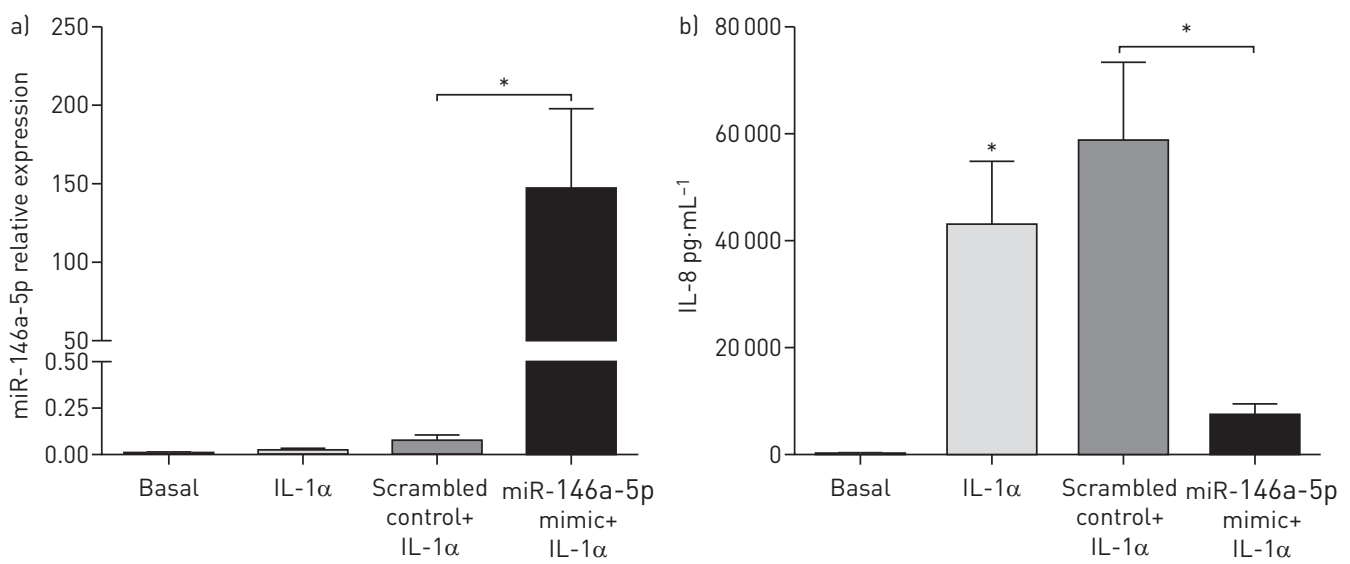

FIGURE 5 miR-146a-5p reduces interleukin (IL)-1 $\alpha$-induced IL-8 release in lung fibroblasts. MRC-5 fibroblasts were seeded and immediately transfected with $25 \mathrm{nM}$ miR-146a-5p mimic or the scrambled control for $48 \mathrm{~h}$. MRC-5 fibroblasts were then serum-deprived overnight and stimulated with/without $1 \mathrm{ng} \cdot \mathrm{mL}^{-1}$ recombinant human IL-1 $\alpha$ for $24 \mathrm{~h}$. a) miR-146a-5p mRNA expression in MRC-5 fibroblasts was related to the housekeeping noncoding RNU48, expressed as $2^{-\Delta C_{t}}$. Data are presented as mean \pm SEM. b) IL-8 release from MRC-5 fibroblasts. Data are presented as mean \pm SEM ( $n=3$ or 4 independent experiments). $*$ : $p<0.05$, between the indicated values.

enhanced release of pro-inflammatory mediators such as IL-8 and IL-6 from lung fibroblasts upon their co-culture [14]. In addition, we showed that IL-1 $\alpha$ was secreted at baseline and was responsible for a pro-inflammatory switch in lung fibroblasts [14].

In the present study, overexpression of miR-146a-5p reduced the IL-1 $\alpha$-induced IL- 8 secretion from lung fibroblasts. Several regulatory mechanisms to modulate the effects of IL- $1 \alpha$ are present in vivo, such as the secretion of the naturally occurring IL-1 receptor antagonist (IL-1Ra) and the decoy IL-1R2 receptor $[27,30]$. Apart from these mechanisms, miR-146a-5p has emerged as a crucial regulator of the IL-1 pathway [25]. In line with previous studies [22, 25], we show that the induction of miR-146a-5p is dependent on IL- $1 \alpha$ stimulation and also causes a downregulation in IRAK-1 protein expression. IRAK-1 is an important serine/threonine kinase that associates with the IL-1R1 receptor complex upon stimulation [31]. This eventually leads to the activation of transcription factors such as activator protein AP-1 and $\mathrm{NF}-\kappa \mathrm{B}$, which subsequently leads to the induction and release of several inflammatory mediators, including IL-8 [31]. Thus, we hypothesise that the IL-1-induced increase in miR-146a-5p acts in a negative feedback loop to regulate the observed lung fibroblast pro-inflammatory activity upon co-culture with AECs. The induction of miR-146a-5p was further enhanced when epithelial cells were pre-stimulated with CSE. This indicates an increased demand for miR-146a-5p induction as a negative feedback mechanism to counteract the enhanced release of IL- $1 \alpha$ from the airway epithelium in smokers.

Of interest, the observed increase in miR-146a-5p expression upon co-culture with epithelial cells was smaller in COPD-derived lung fibroblasts compared with control-derived lung fibroblasts. In line with our previous study [14], this reduced induction of miR-146a-5p may lead to an impaired feedback inhibition of the fibroblast-derived inflammation resulting from a higher production of IL-1 $\alpha$ from COPD-derived epithelium exposed to CSE (figure 6). SATO et al. [12] additionally found less induction of miR-146a-5p in fibroblasts from COPD patients compared with healthy subjects after IL-1 $\beta /$ TNF- $\alpha$ stimulation. This downregulation was associated with increased expression of the cyclooxygenase- 2 enzyme and an increased production of the inflammatory mediator prostaglandin $\mathrm{E}_{2}$ in the sputum of COPD patients [12]. The difference in the induction of miR-146a-5p in PHLFs from our model was only seen after $72 \mathrm{~h}$ of co-culture, but not after the 24-h stimulation of PHLFs with epithelial conditioned medium. This suggests that prolonged periods of exposure to IL- $1 \alpha$ are required to induce differential miR-146a-5p induction between COPD and control-derived fibroblasts which may be representative of the lung epithelial-mesenchymal trophic unit in COPD where there is a chronic exposure to cigarette smoke and the resultant epithelial-derived IL-1 $\alpha[14,19,32]$. This is also in line with PERrY et al. [13], who suggested a time- and concentration-dependent effect of IL-1 on miR-146a-5p expression.

To elucidate the underlying mechanism responsible for the lower induction of miR-146a-5p by COPD-derived PHLFs upon co-culture with epithelial cells, we investigated the possible involvement of RelB, a member of the NF- $\kappa B$ family, which has been shown to regulate miR-146a-5p expression [20]. Although SHERIDAN et al. [33] reported a lower expression of RelB in PHLFs from smokers with and without COPD compared with those from nonsmokers, we did not find a difference in expression in RelB 
a)
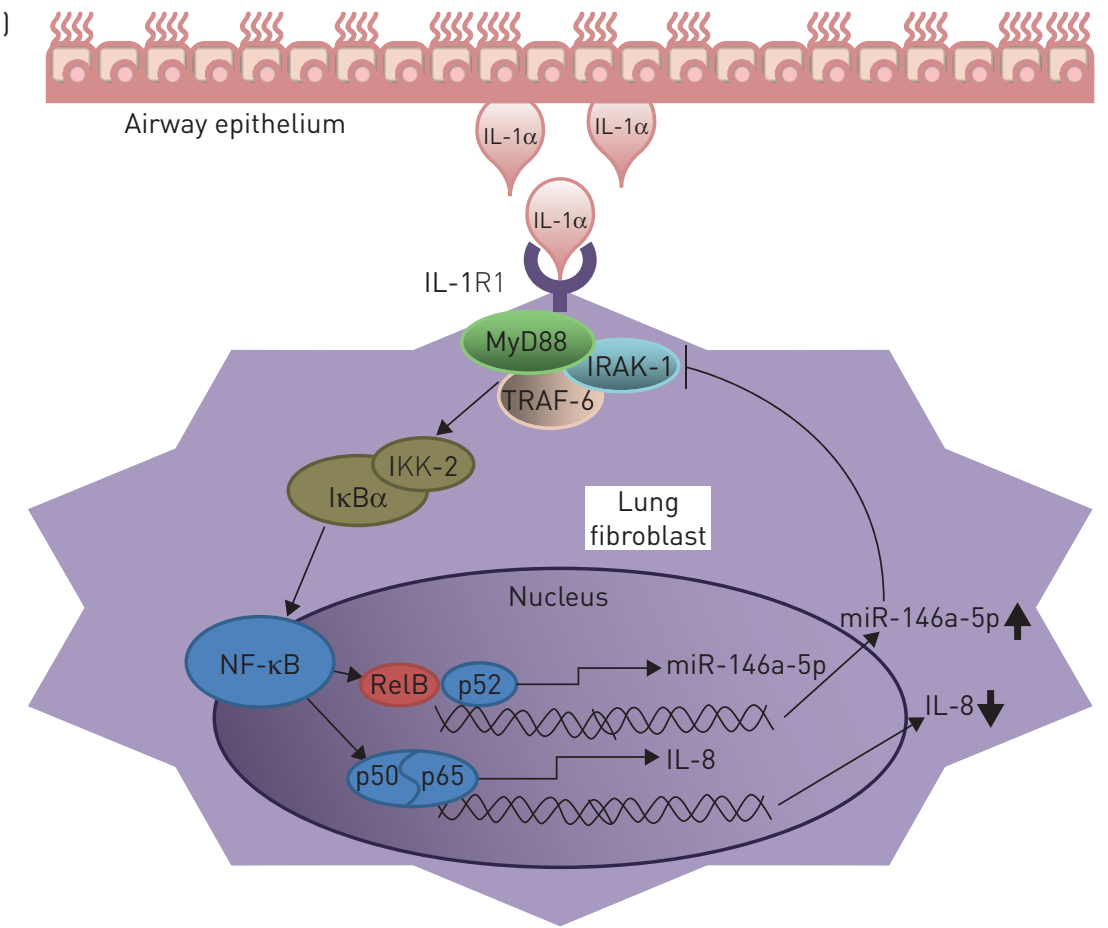

b)

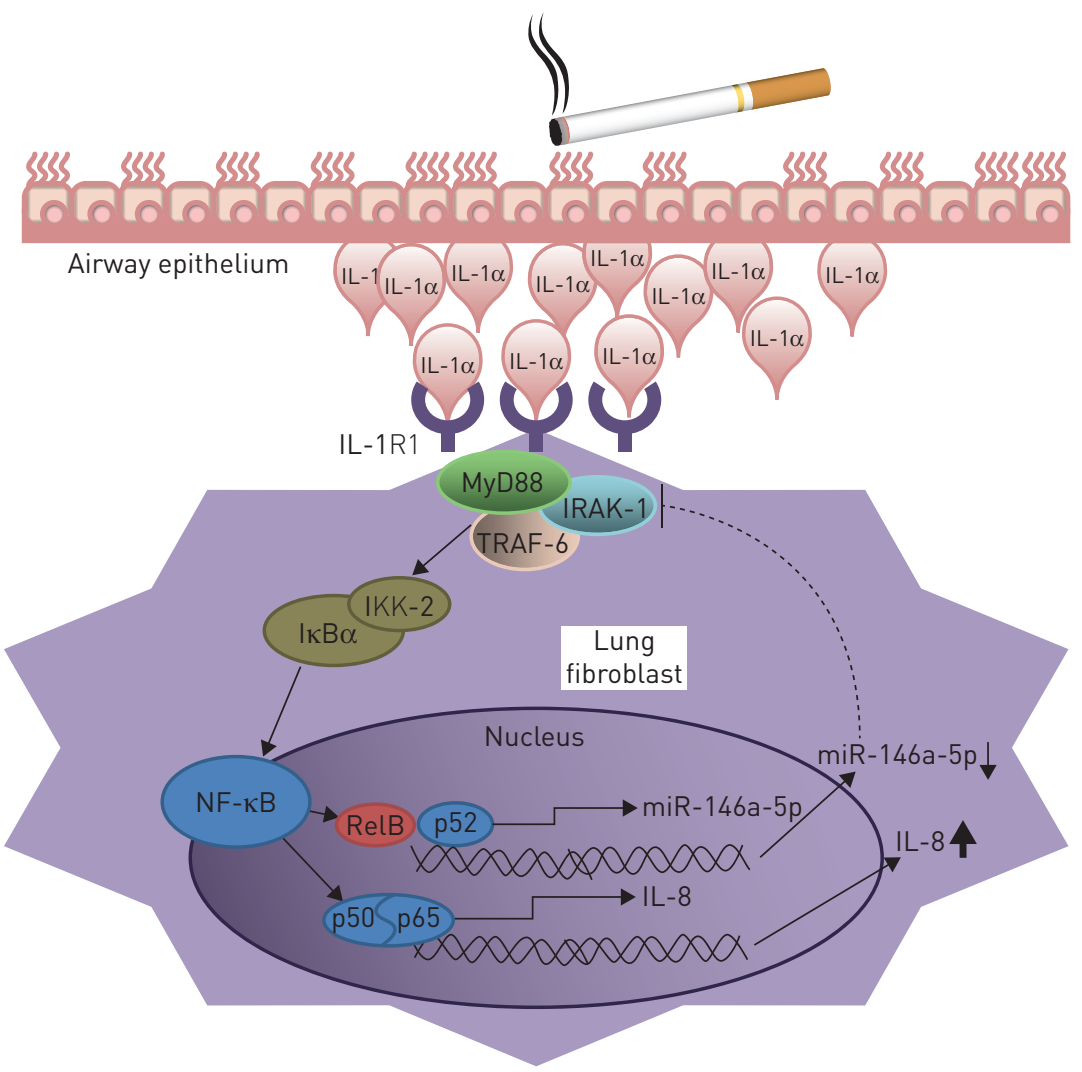

FIGURE 6 Proposed role of miR-146a-5p in the cross-talk between airway epithelial cells (AECs) and lung fibroblasts $[12,14,20,22,24]$. a) In controls, epithelial-derived interleukin (IL)-1 $\alpha$ causes an induction of miR-146a-5p expression as well as release of IL-8 from lung fibroblasts. miR-146a-5p then binds to and downregulates the expression of IL-1 receptor (IL-1R)-associated kinase (IRAK)-1 downstream of the IL-1 pathway in a feedback loop to dampen the NF-KB activation and the inflammatory effects of the epithelial-derived IL-1 $\alpha$ on pulmonary fibroblasts. b) In chronic obstructive pulmonary disease (COPD), cigarette smoke exposure causes an increased IL- $1 \alpha$ release from AECs which further increases IL- 8 release from fibroblasts. However, in COPD-derived fibroblasts, the IL-1 $\alpha$-induced increase in miR-146a-5p expression is lower compared with control-derived fibroblasts, which then contributes to lower feedback inhibition of the NF- $\mathrm{KB}$ activation and an exaggerated pro-inflammatory response. MyD88: myeloid differentiation primary response gene 88; TRAF: tumour necrosis factor receptor-associated factor; IKK: I KB kinase. 
mRNA expression between non-COPD and COPD-derived PHLFs in our co-culture model. A common G>C SNP rs2910164 in the primary miR-146a-5p sequence has been associated with a reduction in the expression of mature miR-146a-5p [21]. Of interest, we found that PHLFs from donors homozygous for the GG allele of SNP rs2910164, which were all but one from COPD patients, had a lower miR-146a-5p induction after co-culture than fibroblasts from donors heterozygous for this allele. This indicates that the expression of this SNP is associated with a lower miR-146a-5p induction in COPD-derived lung fibroblasts in our co-culture model. Of interest, WANG et al. [34] showed that this particular SNP is also associated with a lower miR-146a-5p expression in relation to COPD.

In conclusion, this study demonstrates that the pro-inflammatory phenotype of COPD lung fibroblasts resulting from a dysregulated epithelial-fibroblast interaction in our co-culture model may, at least in part, be due to the reduced ability of COPD-derived fibroblasts to upregulate miR-146a-5p to counter-regulate pro-inflammatory activity. miRNAs are likely to have therapeutic potential [5] with miRNA therapies recently making it through to clinical trials [35]. Hence, our finding could provide a basis for further investigations to target chronic inflammation in COPD.

\section{Acknowledgements}

Author contributions: E.T. Osei, L. Florez-Sampedro, D.S. Postma, W. Timens, T.L. Hackett, I.H. Heijink and C-A. Brandsma were involved in study design and conceptualisation. E.T. Osei, L. Florez-Sampedro, H. Tasena, J.A. Noordhoek and A. Faiz performed experiments. A. Faiz performed SNP analysis. E.T. Osei analysed data from experiments. C-A. Brandsma and I.H. Heijink were involved in supervision of the experiments. E.T. Osei wrote the manuscript. C-A. Brandsma and I.H. Heijink revised the manuscript. D.S. Postma, W. Timens and T.L. Hackett edited the manuscript. The final version was critically reviewed and approved by all authors.

\section{References}

1 Decramer M, Janssens W, Miravitlles M. Chronic obstructive pulmonary disease. Lancet 2012; 379 : $1341-1351$.

2 Hogg JC, Timens W. The pathology of chronic obstructive pulmonary disease. Annu Rev Pathol 2009; 4: 435-459.

3 Løkke A, Lange P, Vestbo J, et al. Udvikling af kronisk obstruktiv lungesygdom - 25-ars-opfolgningsstudie af baggrundsbefolkning. [Developing COPD - 25 years follow-up study of the general population.] Ugeskr Laeg 2006; 168: 4422-4424.

4 Sakao S, Tatsumi K. The importance of epigenetics in the development of chronic obstructive pulmonary disease. Respirology 2011; 16: 1056-1063.

5 Osei ET, Florez-Sampedro L, Timens W, et al. Unravelling the complexity of COPD by microRNAs: it's a small world after all. Eur Respir J 2015; 46: 807-818.

6 Ha M, Kim VN. Regulation of microRNA biogenesis. Nat Rev Mol Cell Biol 2014; 15: 509-524.

7 Ezzie ME, Crawford M, Cho JH, et al. Gene expression networks in COPD: microRNA and mRNA regulation. Thorax 2012; 67: 122-131.

8 Graff JW, Powers LS, Dickson AM, et al. Cigarette smoking decreases global microRNA expression in human alveolar macrophages. PLoS One 2012; 7: e44066.

9 Bosse Y, Postma DS, Sin DD, et al. Molecular signature of smoking in human lung tissues. Cancer Res 2012; 72: 3753-3763.

10 Akbas F, Coskunpinar E, Aynaci E, et al. Analysis of serum micro-RNAs as potential biomarker in chronic obstructive pulmonary disease. Exp Lung Res 2012; 38: 286-294.

11 Van Pottelberge GR, Mestdagh P, Bracke KR, et al. MicroRNA expression in induced sputum of smokers and patients with chronic obstructive pulmonary disease. Am J Respir Crit Care Med 2011; 183: 898-906.

12 Sato T, Liu X, Nelson A, et al. Reduced miR-146a increases prostaglandin $\mathrm{E}_{2}$ in chronic obstructive pulmonary disease fibroblasts. Am J Respir Crit Care Med 2010; 182: 1020-1029.

13 Perry MM, Moschos SA, Williams AE, et al. Rapid changes in microRNA-146a expression negatively regulate the IL-1beta-induced inflammatory response in human lung alveolar epithelial cells. J Immunol 2008; 180: 5689-5698.

14 Osei ET, Noordhoek JA, Hackett TL, et al. Interleukin-1 $\alpha$ drives the dysfunctional cross-talk of the airway epithelium and lung fibroblasts in COPD. Eur Respir J 2016; 48: 350-358.

15 Heijink IH, Kies PM, Kauffman HF, et al. Down-regulation of E-cadherin in human bronchial epithelial cells leads to epidermal growth factor receptor-dependent Th2 cell-promoting activity. J Immunol 2007; 178: 7678-7685.

16 Noordhoek JA, Postma DS, Chong LL, et al. Different modulation of decorin production by lung fibroblasts from patients with mild and severe emphysema. COPD 2005; 2: 17-25.

17 Brandsma CA, Timens W, Jonker MR, et al. Differential effects of fluticasone on extracellular matrix production by airway and parenchymal fibroblasts in severe COPD. Am J Physiol Lung Cell Mol Physiol 2013; 305: L582-L589.

18 Slebos DJ, Ryter SW, van der Toorn M, et al. Mitochondrial localization and function of heme oxygenase-1 in cigarette smoke-induced cell death. Am J Respir Cell Mol Biol 2007; 36: 409-417.

19 Botelho MF, Bauer CM, Finch D, et al. IL-1 $\alpha /$ IL1-R1 expression in chronic obstructive pulmonary disease and mechanistic relevance to smoke-induced neutrophilia in mice. PLoS One 2011; 6: e28457-e28457.

20 Zago M, Rico de Souza A, Hecht E, et al. The NF- $\kappa$ B family member RelB regulates microRNA miR-146a to suppress cigarette smoke-induced COX-2 protein expression in lung fibroblasts. Toxicol Lett 2014; 226 : 107-116.

21 Jazdzewski K, Murray EL, Franssila K, et al. Common SNP in pre-miR-146a decreases mature miR expression and predisposes to papillary thyroid carcinoma. Proc Natl Acad Sci USA 2008; 105: 7269-7274.

22 Jiang W, Kong L, Ni Q, et al. miR-146a ameliorates liver ischemia/reperfusion injury by suppressing IRAK1 and TRAF6. PLoS One 2014; 9: e101530.

23 Rusca N, Monticelli S. MiR-146a in immunity and disease. Mol Biol Int 2011; 2011: 437301. 
24 Perry MM, Williams AE, Tsitsiou E, et al. Divergent intracellular pathways regulate interleukin-1beta-induced miR-146a and miR-146b expression and chemokine release in human alveolar epithelial cells. FEBS Lett 2009; 583: 3349-3355.

25 Bhaumik D, Scott GK, Schokrpur S, et al. MicroRNAs miR-146a/b negatively modulate the senescence-associated inflammatory mediators IL-6 and IL-8. Aging 2009; 1: 402-411.

26 Tuder RM, Kern JA, Miller YE. Senescence in chronic obstructive pulmonary disease. Proc Am Thorac Soc 2012; 9: 62-63.

27 Garlanda C, Dinarello CA, Mantovani A. The interleukin-1 family: back to the future. Immunity 2013; 39: 1003-1018.

28 Martin TR, Frevert CW. Innate immunity in the lungs. Proc Am Thorac Soc 2005; 2: 403-411.

29 Pauwels NS, Bracke KR, Dupont LL, et al. Role of IL- $1 \alpha$ and the Nlrp3/caspase-1/IL-1 $\beta$ axis in cigarette smoke-induced pulmonary inflammation and COPD. Eur Respir J 2011; 38: 1019-1028.

30 Arend WP. The balance between IL-1 and IL-1Ra in disease. Cytokine Growth Factor Rev 2002; 13: 323-340.

31 Jain A, Kaczanowska S, Davila E. IL-1 Receptor-associated kinase signaling and its role in inflammation, cancer progression, and therapy resistance. Front Immunol 2014; 5: 553.

32 Suwara MI, Green NJ, Borthwick LA, et al. IL-1 $\alpha$ released from damaged epithelial cells is sufficient and essential to trigger inflammatory responses in human lung fibroblasts. Mucosal Immunol 2014; 7: 684-693.

33 Sheridan JA, Zago M, Nair P, et al. Decreased expression of the NF- $\mathrm{kB}$ family member RelB in lung fibroblasts from smokers with and without COPD potentiates cigarette smoke-induced COX-2 expression. Respir Res 2015; 16: 54 .

34 Wang R, Li M, Zhou S, et al. Effect of a single nucleotide polymorphism in miR-146a on COX-2 protein expression and lung function in smokers with chronic obstructive pulmonary disease. Int J Chron Obstruct Pulmon Dis 2015; 10: 463-473.

35 Janssen HL, Reesink HW, Lawitz EJ, et al. Treatment of HCV infection by targeting microRNA. N Engl J Med 2013; 368: 1685-1694. 